\title{
Distribution and Drug Susceptibility of Candida spp. Associated With Female Genital Tract Infection, Chongqing, China
}

\author{
Xiaodong Luo, ${ }^{1, *}$ Xiaojing Dong, ${ }^{1}$ and Zhi Pen ${ }^{1}$ \\ ${ }^{1}$ Department of Obstetrics and Gynecology, Second Affiliate Hospital, Chongqing University of Medical Sciences, Chongqing, China \\ "Corresponding author: Xiaodong Luo, Department of Obstetrics and Gynecology, the Second Affiliate Hospital, Chongqing University of Medical Sciences, Chongqing, China. \\ Tel: +86-2363693484, Fax: +86-2363693484, E-mail: ald735@163.com
}

Received 2014 July 23; Revised 2015 February 09; Accepted 2015 March 04.

\begin{abstract}
Background: Vulvovaginal candidiasis is defined as vulvovaginitis associated with vaginal carriage of Candida spp. and is a common problem with a high rate of morbidity.

Objectives: To investigate the distribution of Candida spp. and evaluate the corresponding antifungal susceptibility in women with genital tract infection in Chongqing, southwestern China.

Patients and Methods: Samples $(\mathrm{n}=2.129)$ were obtained from female patients with symptoms of genital tract infection. Candida spp. were isolated from the specimens and were identified using a coloration medium and the VITEK 2 Compact automatic microbial identification system. Antifungal susceptibility testing was performed using the ATB FUNGUS drug susceptibility testing system.

Results: From 2,129 samples, 478 (22.45\%) isolates of Candida were isolated, of which 395 (82.64\%) were Candida albicans, 39 (8.16\%) were C. glabrata, 21 (4.39\%) were C. tropicalis, 9 (1.88\%) were C. parapsilosis, and 14 (2.93\%) were other Candida spp. The resistance of C. albicans, C. glabrata, and C. tropicalis to 5 antifungal drugs (amphotericin B, voriconazole, fluconazole, 5 -fluorocytosine, and itraconazole) ranged from $0.5 \%$ to $6.4 \%, 0 \%$ to $7.7 \%$, and $0 \%$ to $9.6 \%$, respectively.

Conclusions: Candida albicans was the major pathogen associated with candidiasis of the female genital tract in patients in Chongqing. The results of the antifungal sensitivity of the isolates suggest that it is important for clinicians to administer appropriate antifungals for the treatment of Candida spp. infections.
\end{abstract}

Keywords: Genital Tract Infection, Antifungals, Candida spp

\section{Background}

Vulvovaginal candidiasis is defined as vulvovaginitis associated with vaginal carriage of Candida spp. and is a common problem with a high rate of morbidity. This infection is characterized by vulvar pruritus and increased vaginal discharge. At present, clinical treatment for vulvovaginal candidiasis consists of systemic and/or local use of imidazole-based antifungals (1).

Vaginal infection in pregnant women increases the incidence of fungal dermatitis and thrush in newborns, following vaginal delivery. Some fungal pathogens can move up the reproductive tract and penetrate the fetal membrane, leading to infection, which can result in abortion and/or premature delivery. The diagnosis and treatment of vulvovaginal candidiasis are subject to certain restrictions owing to the long period of time required for fungal culture and identification. In recent years, as a result of increasing sexual activity, the wide use of broad-spectrum antibiotics, anticancer drugs, immunosuppressants, corticosteroids, and oral contraceptives and an increase in the diabetes rate, the incidence of vaginitis associated with Candida spp. has increased significantly. An increase in the antifungal drug resistance in Candida spp. has also been observed, making treatment of this infection more difficult $(2,3)$. Some studies have identified alternative anti- fungal drugs that have been reported to be effective in the treatment of Candida infections. For example, Sadeghi Nejad et al. (4) showed that the extracts from fruits of Heracleum persicum had potential anti-Candida activity.

The emergence of antifungal resistance has resulted in an urgent need for laboratory support in the treatment of fungal infections, which includes the rapid identification of fungal pathogens and selection of appropriate methods for the in vitro susceptibility testing of clinical isolates. Shokohi et al. (5) determined the antifungal susceptibility of Candida spp. collected from oropharyngeal lesions in cancer patients.

\section{Objectives}

The aim of this retrospective study was to investigate recent Candida spp. infections in the genital tracts of female patients in Chongqing and to evaluate the resistance to commonly used antifungals by retrospectively analyzing the composition and drug susceptibility of Candida spp. isolated from the vaginal and cervical secretion specimens in both gynecological outpatients and inpatients between January 2010 and December 2011. 


\section{Patients and Methods}

\subsection{Subjects}

From the outpatients and inpatients at the second affiliate hospital, Chongqing University of Medical Sciences, 2,129 women who presented with increased vaginal discharge or pus, vulvar itching, abdominal pain, and other symptoms of infection of the reproductive tract were selected. Patients from the Chongqing region were selected over a 2-year period from January 2010 to December 2011. This study was performed in accordance with the principles of the Helsinki declaration. ethical approval was obtained from the second affiliate hospital of the Chongqing University of Medical Sciences. The subjects were between 18 and 79 years of age (average age, 35.8 years), and of these subjects, 116 were $<20$ years old, 1,815 were between 20 and 49 years old, and 198 were $\geq 50$ years old. Thirty subjects were unmarried. Patients who had undergone a general physical or gynecological examination for any reason and had used medication within 1 week before specimen collection were excluded from the study.

\subsection{Specimen Collection}

All specimens were collected by qualified gynecologists who obtained vaginal swabs from the dome and sidewalls of the vagina. Vaginal secretions were collected in sterile test tubes containing $1 \mathrm{~mL}$ of $0.9 \%$ saline for the culture of fungi. If the subject was unmarried, the sample was obtained from the vaginal orifice.

\subsection{Culture and Identification of Candida spp}

Vaginal secretions were cultured on Sabouraud dextrose agar (Oxoid, UK) at both $25^{\circ} \mathrm{C}$ and $37^{\circ} \mathrm{C}$ for 2 or 3 days, respectively. Putative positive colonies were white or cream in color with glossy and smooth surfaces. If no colonies were evident within 2 weeks, the sample was considered negative. Yeast-like colonies were Gram-stained to exclude any bacterial colonies that had been mistakenly identified as yeast. Colonies were then inoculated on CHROMagar medium (France) at $35^{\circ} \mathrm{C}$ for $24-48$ hours. Green or emerald green colonies were identified as C. albicans, blue-gray colonies were identified as C. tropicalis, and purple-embossed colonies with smooth and glossy surfaces were identified as C. glabrata. Pink-to-purple large flat colonies with rough edges were identified as C. $\mathrm{kru}$ sei, while colonies of the other phenotypes were identified as other Candida spp. These atypical isolates were further identified using the VITEK 2 compact automatic microbial identification system (bioMerieux, France).

\subsection{Drug Susceptibility Test}

Drug susceptibility testing was performed using the ATB FUNGUS drug susceptibility testing system (bioMerieux company, France). The following drugs and concentrations were tested: 5-flucytosine (0.125 - 64 $\mu \mathrm{g} / \mathrm{mL}$ ); amphotericin B, voriconazole, itraconazole (each between 0.03 and $16 \mu \mathrm{g} / \mathrm{mL}$ ); and fluconazole (0.125 - 64 $\mu \mathrm{g} / \mathrm{mL})$. Testing was performed at $35^{\circ} \mathrm{C}$ for 24 hours. The minimum inhibitory concentrations of all drugs were obtained according to the CLSI M27-A3 guidelines (6, 7). In each test, the reference strain C. albicans ATCC 14053 was used for quality control.

According to the manufacturer instructions, a suspension of C. albicans with a turbidity of 2 McFarland was prepared, and $20 \mu \mathrm{L}$ of this suspension was transferred to an ampule of ATB FUNGUS 3 medium. Then, $135 \mu \mathrm{L}$ of the inoculated medium was transferred into each cupule. After incubation at $35^{\circ} \mathrm{C}$ for 24 hours, the strips were read using the ATB expression bacteriology analyzer automatic system (bioMerieux, France).

\subsection{Statistical Analysis}

Statistical analyses were performed using the SPSS software, version 17.0 (SPSS; Chicago, IL, USA). Data are presented as percentages. All data were compared and analyzed using the chi-square test. $\mathrm{P}<0.05$ were considered statistically significant.

\section{Results}

\subsection{Detection and Distribution of Candida spp}

From 2,129 samples of female genital tract secretions collected over a 2-year period, 478 isolates (positivity rate of $22.45 \%$ ) representing 8 Candida spp. were detected. Of the isolates, $82.64 \%$ were C. albicans, $8.16 \%$ were C. tropicalis, $4.39 \%$ were C. glabrata, $1.88 \%$ were C. parapsilosis, $1.26 \%$ were C. krusei, $1.05 \%$ were C. guilliermondii, $0.42 \%$ were $C$. lusitaniae, and $0.21 \%$ were C. dubliniensis (Table 1 ).

\subsection{Detection Rate of Candidiasis in Patients in Different Age Groups}

According to the world health organization (WHO) standards and the age demographics in China, patients were divided into three groups: (1) subjects $<20$ years of age, (2) subjects between 20 and 49 years of age, and (3) subjects $>50$ of age. The results showed that the detection rates of Candida-related vaginal disease in age groups 1 and 2 were significantly higher than those in age group 3 ( $\mathrm{P}<$ 0.05). Differences between age groups 1 and 2 were not statistically significant $(\mathrm{P}>0.05)$, as shown in Table 2 . 
Table 1. Distribution of the Detected Candida spp $^{\mathrm{a}}$

\begin{tabular}{lc}
\hline Species & Number of Strains \\
\hline C. albicans & $395(82.64)$ \\
\hline C.glabrata & $39(8.16)$ \\
\hline C. tropicalis & $21(4.39)$ \\
\hline C. parapsilosis & $9(1.88)$ \\
\hline C. krusei & $6(1.26)$ \\
\hline C. guilliermondii & $5(1.05)$ \\
\hline C. lusitaniae & $2(0.42)$ \\
\hline C. dubliniensis & $1(0.21)$ \\
\hline
\end{tabular}

${ }^{\mathrm{a}}$ Values are expressed as No. (\%).

\subsection{Antifungal Sensitivity Test}

Resistance of the 3 most commonly isolated Candida spp. to the 5 antifungals tested was low (Table 3$)$. The resistance rate to amphotericin B was the lowest ( 0 - $0.5 \%$ ) with sensitivity rates between $97.4 \%$ and $100 \%$. The resistance rates to voriconazole were between $0 \%$ and $5.2 \%$ and the sensitivity rates were between $89.7 \%$ and $100 \%$, while the resistance rates to itraconazole were between $0 \%$ and $7.7 \%$ and the sensitivity rates were between $82.1 \%$ and $90.4 \%$.

\section{Discussion}

Vulvovaginal candidiasis frequently colonizes the female reproductive tract with typical symptoms of vaginal itching, redness, and tofu-like vaginal discharge (8). Owing to the inappropriate use of antifungal drugs (i.e., repeated administration or long-term use), the appearance of drugresistant strains has been increasing in China, making antifungal treatment increasingly difficult (9).

In this study, 2.129 vaginal secretions from patients with reproductive tract infections in the Chongqing region were analyzed for fungal infection. The results showed that 478 subjects were infected with 8 Candida spp., of which C. albicans accounted for 395 cases, C. glabrata accounted for 39 cases, C. tropicalis accounted for 21 cases, and other Candida accounted for 23 cases. This indicates that Candida infection is high in patients with gynecological reproductive tract infections in the Chongqing region.

Colonization by Candida spp. is affected by the immune status of the host, lower body resistance, $\mathrm{pH}$ changes in the vagina, lifestyle, abuse of antibiotics and pregnancy $(10,11)$. In our study, the overall positivity rate of Candida infection was $22.45 \%$, of which C. albicans was the main species identified. This is consistent with the results of the other studies (12-16). Candida glabrata and C. tropicalis were the next commonly detected species. This observation differs somewhat from the other studies, where C. krusei was more commonly isolated than C. tropicalis, but these variations may be owing to differences in the populations and geographical locations of the studies. Recent reports indicate that the association of C. glabrata with vaginal candidiasis has been gradually increasing (15), and this may be related to the wide use of clinical azole drugs or nonstandardized treatments.

Even if there is no direct evidence that vulvovaginal candidiasis is sexually transmitted, the incidence of vulvovaginal candidiasis is known to increase upon initiation of sexual activity (17). Indeed, sexual transmission between partners may be a factor in the increasing incidence of candidiasis. Studies have shown that the infection rates of spouses of Candida spp.-positive male partners were 4 times higher than those of male partners without Candida infection. In addition, Candida spp. infections were detected in the penis of $15 \%$ of the partners of female patients with candidiasis $(18,19)$. Our study has shown that the detection rates of Candida spp. in the under 20 and 20 49 years age groups were significantly higher than in the group of patients $>50$ years. This indicates that vaginal candidiasis occurs more often in women of reproductive age in Chongqing. We believe that the increase in vaginal infections in this population may be related to their more active sexual behavior.

In recent years, drug resistance in Candida spp. to common antifungal drugs has been increasing $(20,21)$. In our study, drug susceptibility tests were performed on 478 strains of Candida. Our results showed that 3 main species, including C. albicans, had low resistance to the 5 antifungal drugs. Resistance rates were $<8 \%$, while the sensitivity rates were $>80 \%$. In particular, resistance rates to amphotericin B were the lowest, ranging from $0 \%$ to $0.5 \%$, with sensitivity rates ranging from $97.4 \%$ to $100 \%$; resistance rates to voriconazole ranged from $0 \%$ to $5.2 \%$ and sensitivity rates ranged from $89.7 \%$ to $100 \%$. However, owing to the side effects of amphotericin B and voriconazole, their use in candidiasis treatment is limited. The sensitivity rates of C. glabrata to fluconazole and itraconazole were $84.6 \%$ and $82.1 \%$, respectively, which were lower than those of $C$. tropicalis and C. albicans.

Buitron Garcia-Figueroa et al. (22) reported that in vaginal candidiasis, the infection rates of C. glabrata is rising commensurate with the drug resistance rates to fluconazole (68.2\%). Studies by Peman et al. (23) also showed that the overall susceptibility rates for itraconazole and fluconazole were $77.6 \%$ and $91.9 \%$, respectively. Resistance rates were only observed in C. glabrata for itraconazole (24.1\%) and posaconazole (14.5\%), and in C. krusei for itraconazole (81.5\%). In contrast Gualco et al. (24) reported that 
Table 2. Detection Rates of Vaginitis in Women of Different Ages ${ }^{\mathrm{a}}$

\begin{tabular}{|c|c|c|c|c|}
\hline Age Group, y & Number of Cases & Candida & C. albicans & Other Candida spp \\
\hline$<\mathbf{2 0}$ & 116 & $26(22.4)$ & $20(76.9)$ & $6(23.1)$ \\
\hline $20-49$ & 1815 & $429(23.6)$ & $357(83.2)$ & $72(16.8)$ \\
\hline$>49$ & 198 & $23(11.6)$ & $18(78.3)$ & $5(21.7)$ \\
\hline Total & 2129 & 478 & 395 & 83 \\
\hline
\end{tabular}

${ }^{\mathrm{a}}$ Values are expressed as No. (\%).

Table 3. Drug Susceptibility in the Most Prevalent Candida spp $^{\mathrm{a}}$

\begin{tabular}{|c|c|c|c|c|c|c|c|c|c|}
\hline \multirow[t]{2}{*}{ Antifungals } & \multicolumn{3}{|c|}{ C. albicans (395 Isolates) } & \multicolumn{3}{|c|}{ C. glabrata (39 Isolates) } & \multicolumn{3}{|c|}{ C. tropicalis (21 Isolates) } \\
\hline & $\mathbf{R}$ & I & s & $\mathbf{R}$ & I & s & $\mathbf{R}$ & I & s \\
\hline Amphotericin & 0.5 & 0.5 & 99.0 & 0.0 & 2.6 & 97.4 & 0.0 & 0.0 & 100.0 \\
\hline Voriconazole & 1.7 & 5.1 & 93.2 & 5.2 & 5.1 & 89.7 .5 & 0.0 & 0.0 & 100.0 \\
\hline Fluconazole & 5.3 & 4.6 & 90.1 & 7.7 & 7.7 & 84.6 & 0.0 & 4.8 & 95.2 \\
\hline 5-Florocytosineosinosine & 3.1 & 6.3 & 90.6 & 5.1 & 2.6 & 92.3 & 4.8 & 0.0 & 95.2 \\
\hline Itraconazole & 6.4 & 7.8 & 85.8 & 7.7 & 10.2 & 82.1 & 4.8 & 4.8 & 90.4 \\
\hline
\end{tabular}

${ }^{\mathrm{a}}$ Values are expressed as percentages.

the resistance rates of $C$. albicans to fluconazole and itraconazole were $0.7 \%$ and $2.7 \%$, respectively. The different resistance rates observed in these studies may be owing to the differences in the study populations and geographical locations or differences in antifungal use, which is not regulated in China.

On the basis of our results, we suggest that the identification of yeast infections by smear microscopy alone is not sufficient and that clinicians should attempt to cultivate causative Candida spp. from vaginal secretions and design antifungal treatments based on drug susceptibility testing (25). This approach should help to reduce the recurrence of Candida infections and to mitigate against increasing antifungal resistance.

\section{Acknowledgments}

The authors are grateful to Yonghong Zhang and the department of clinical and gynecology laboratory, the second affiliate hospital, Chongqing University of Medical Sciences. The authors declare no conflict of interest.

\section{Footnote}

Authors' Contribution: Xiaodong Luo conducted the specimen studies, participated in the susceptibility testing, and drafted the manuscript; Xiaojing Dong contributed to the study design and helped to draft the manuscript; Zhi Pen conceived of the study, contributed to the study design and coordination, and performed the statistical analysis. All authors read and approved the final manuscript.

\section{References}

1. Nurbhai M, Grimshaw J, Watson M, Bond CM, Mollison JA, Ludbrook A. Oral versus intra-vaginal imidazole and triazole anti-fungal treatment of uncomplicated vulvovaginal candidiasis (thrush). Cochrane Libr. 2007;17(4):CD002845.

2. Inigo M, Peman J, Del Pozo JL. Antifungal activity against Candida biofilms. Int J Artif Organs. 2012;35(10):780-91. doi: 10.5301/ijao.5000170. [PubMed: 23138707].

3. Taff HT, Mitchell KF, Edward JA, Andes DR. Mechanisms of Candida biofilm drug resistance. Future Microbiol. 2013;8(10):1325-37. doi: 10.2217/fmb.13.101. [PubMed: 24059922].

4. Sadeghi Nejad B, Rajabi M, Zarei Mamoudabadi A, Zarrin M. In vitro anti-candida activity of the hydroalcoholic extracts of heracleum persicum fruit against phatogenic candida species. Jundishapur J Microbiol. 2014;7(1):e8703. doi: 10.5812/jjm.8703. [PubMed: 25147655].

5. Shokohi T, Bandalizadeh Z, Hedayati MT, Mayahi S. In vitro antifungal susceptibility of Candida species isolated from oropharyngeal lesions of patients with cancer to some antifungal agents. Jundishapur JMicrobiol. 2011;4(2):19-26.

6. Clinical and Laboratory Standards Institute . Reference method for broth dilution antifungal susceptibility testing of yeasts; Approved standard M27-A3. 3 ed. Wayne: Clinical and Laboratory Standards Institute; 2008.

7. Clinical and laboratory standard institute . reference method for broth dilution antifungal susceptibility testing of yeasts; approved standard- third edition M27-S3. Wayne, Pennsylvania, USA: National Committee for Clinical Laboratory Standards; 2008.

8. Mendling W, Brasch J. Guideline vulvovaginal candidosis (2010) of the german society for gynecology and obstetrics, the working group for infections and infectimmunology in gynecology and obstetrics, the german society of dermatology, the board of german dermatologists and the german speaking mycological society. 2012. 
9. Wang Y, Huang H, LI J, Wen XD, Jiang YY. An update on drug resistance of fungal pathogens. Pharm Care and Res. 2009;3:005.

10. Rathod SD, Buffler PA. Highly-cited estimates of the cumulative incidence and recurrence of vulvovaginal candidiasis are inadequately documented. BMC Womens Health. 2014;14(1):43. doi: 10.1186/14726874-14-43. [PubMed: 24612727].

11. Filioti J, Spiroglou K, Panteliadis CP, Roilides E. Invasive candidiasis in pediatric intensive care patients: epidemiology, risk factors, management, and outcome. Intensive Care Med. 2007;33(7):1272-83. doi: 10.1007/s00134-007-0672-5. [PubMed: 17503015].

12. Liu XP, Fan SR, Peng YT, Zhang HP. Species distribution and susceptibility of Candida isolates from patient with vulvovaginal candidiasis in Southern China from 2003 to 2012. J Mycol Med. 2014;24(2):106-11 doi: 10.1016/j.mycmed.2014.01.060. [PubMed: 24746725].

13. Xu QR, Yan L, Lv QZ, Zhou M, Sui X, Cao YB, et al. Molecular genetic techniques for gene manipulation in Candida albicans. Virulence. 2014;5(4):507-20. doi: 10.4161/viru.28893. [PubMed: 24759671].

14. Vijaya D, Dhanalakshmi TA, Kulkarni S. Changing trends of vulvovaginal candidiasis. J Lab Physicians. 2014;6(1):28-30. doi: 10.4103/09742727.129087. [PubMed: 24696557].

15. Mahmoudi Rad M, Zafarghandi A, Amel Zabihi M, Tavallaee M, Mirdamadi Y. Identification of Candida species associated with vulvovaginal candidiasis by multiplex PCR. Infect Dis Obstet Gynecol. 2012;2012:872169. doi: 10.1155/2012/872169. [PubMed: 22791958].

16. Li J, Fan SR, Liu XP, Li DM, Nie ZH, Li F, et al. Biased genotype distributions of Candida albicans strains associated with vulvovaginal candidosis and candidal balanoposthitis in China. Clin Infect Dis 2008;47(9):1119-25. doi:10.1086/592249. [PubMed: 18808351].

17. Spence D. Candidiasis (vulvovaginal). BMJ Clin Evid. 2010 Jan 5;2010. [PubMed: 21718579].
18. Le J. Obstetrics and gynecology [in Chinese]. 6 ed. Beijing: People's medical publishing house; 2004

19. Linhares LM, Witkin SS, Miranda SD, Fonseca AM, Pinotti JA, Ledger WJ. Differentiation between women with vulvovaginal symptoms who are positive or negative for Candida species by culture. Infect Dis Obstet Gynecol. 2001;9(4):221-5. doi: 10.1155/S1064744901000369. [PubMed: 11916179].

20. Nett JE. Future directions for anti-biofilm therapeutics targeting Candida. Expert Rev Anti Infect Ther. 2014;12(3):375-82. doi: 10.1586/14787210.2014.885838. [PubMed: 24506174].

21. Hsu CC, Lai WL, Chuang KC, Lee MH, Tsai YC. The inhibitory activity of linalool against the filamentous growth and biofilm formation in Candida albicans. Med Mycol. 2013;51(5):473-82. doi: 10.3109/13693786.2012.743051. [PubMed: 23210679].

22. Buitron Garcia-Figueroa R, Araiza-Santibanez J, Basurto-Kuba E, Bonifaz-Trujillo A. Candida glabrata: an emergent opportunist in vulvovaginitis. Cir Cir. 2009;77(6):423-7. [PubMed: 20433786].

23. Peman J, Canton E, Quindos G, Eraso E, Alcoba J, Guinea J, et al. Epidemiology, species distribution and in vitro antifungal susceptibility of fungaemia in a Spanish multicentre prospective survey. J Antimicrob Chemother. 2012;67(5):1181-7. doi: 10.1093/jac/dks019. [PubMed: 22351683].

24. Gualco L, Debbia EA, Bandettini R, Pescetto L, Cavallero A, Ossi MC, et al. Antifungal resistance in Candida spp. isolated in Italy between 2002 and 2005 from children and adults. Int J Antimicrob Agents. 2007;29(2):179-84. doi: 10.1016/j.ijantimicag.2006.08.047. [PubMed: 17175140].

25. Diba K, Namaki A, Ayatolahi H, Hanifian H. Rapid identification of drug resistant Candida species causing recurrent vulvovaginal candidiasis. Med Mycol J. 2012;53(3):193-8. [PubMed: 23149354]. 